\title{
When do irrelevant visual stimuli impair processing of identical targets?
}

\author{
PETER WÜHR \\ Friedrich Alexander University, Erlangen, Germany \\ and \\ JOCHEN MÜSSELER \\ Max Planck Institute for Human Cognitive and Brain Sciences, Munich, Germany
}

\begin{abstract}
Three experiments investigated whether the repeated-letter inferiority effect (RLIE) and repetition blindness (RB) are identical phenomena or not and how the RLIE can be reconciled with the flanker compatibility effect (FCE). Participants reported a masked target and ignored an unmasked distractor. We manipulated the type of distractor (identical, alternative target, or neutral), the order of presenting distractor and target, and the predictability of target location. When distractors preceded the targets, distractors identical to the target always caused deficits in target processing (i.e., RB). With simultaneous presentation, identical distractors caused deficits (i.e., an RLIE) for unpredictable target locations only. Yet the RLIE was significantly smaller than RB. This result suggests that simultaneously presented stimuli are identified serially, in random order, if target position is unpredictable. As a result, $\mathrm{RB}$ arises only in the $50 \%$ of all trials with identical distractors in which the distractor is identified before the target. With simultaneous presentation and predictable target location, however, parallel processing of targets and distractors was possible that gave rise to an FCE in recognition accuracy. Analyses of false alarm rates revealed no evidence of significant response biases.
\end{abstract}

An informative tool for investigating visual perception in humans has been to study the effects of identical distractors (i.e., not-to-be-reported stimuli that are identical to the target) on processing of a target stimulus (i.e., a tobe-reported stimulus). Such investigations have discovered many interesting phenomena, and to explain them, researchers have suggested many functional mechanisms of visual processing. Among these phenomena are the repeated-letter inferiority effect (RLIE; Egeth \& Santee, 1981), repetition blindness (RB; Kanwisher, 1987), redundancy gain (RG; Miller, 1982), and the flanker compatibility effect (FCE; B. A. Eriksen \& C. W. Eriksen, 1974). On the other hand, similar experimental situations have produced conflicting empirical observations (e.g., RLIE vs. RG). Moreover, different explanations have been proposed to explain similar phenomena that occur in different situations (e.g., RB vs. RLIE). The goal of the present study is to resolve at least some of these empirical and theoretical discrepancies. In particular, the study addresses discrepancies between the RLIE and the phenomena of RB, RG, and FCE. In the following sec-

J.M. is now at the Institut für Arbeitsphysiologie an der Universität Dortmund, Dortmund, Germany. The authors thank Silvia Bauer, Veronika Gärtner, Mathias Küfner, Renate Tschakert, Manuela Scherer, Anja Kriegs, and Diana Berari for collecting parts of the data. Moreover, we are grateful to Tracy Brown, John Flowers, and Lisa Fournier for their constructive discussion of a previous manuscript. Correspondence relating to this article may be sent to P. Wühr, Institut für Psychologie, Universität Erlangen, Kochstraße 4, 91054 Erlangen, Germany (e-mail: prwuehr@phil.uni-erlangen.de). tions, the phenomena and the pairwise discrepancies among them are described, and a theoretical solution for these discrepancies is presented. Finally, three experiments are reported that tested the new account.

\section{Repeated-Letter Inferiority Effect}

Bjork and Murray (1977) investigated the effects of simultaneously presented identical and nonidentical distractors in a visual display on the accuracy of identifying a target. Participants observed two simultaneously presented letters that were preceded and followed by a mask. The letters appeared in different columns of a $4 \times 4$ matrix, and a postcue indicated the column that had to be responded to. Participants then decided which of two letters specified in advance had been presented in the cued column. The result was that repeated letters in the display decreased identification accuracy for the target. More precisely, identification performance was impaired with an identical distractor when compared with performance with a neutral distractor (i.e., a letter never presented as a target). A further result was that an incompatible distractor (i.e., the alternative target) allowed for performance similar to that with a neutral distractor. Subsequent studies have replicated and extended this finding (Egeth \& Santee, 1981; Keren \& Boer, 1985; Santee \& Egeth, 1980, 1982).

Bjork and Murray (1977) explained this RLIE by assuming interactions between early visual processing channels (cf. Estes, 1972, 1974). They proposed that the presence of a particular visual feature activates an input channel 
that leads to a corresponding feature detector (or code). Furthermore, excitation of a channel was assumed to inhibit other channels - particularly those leading to the same feature detector. In other words, identical distractors impair target processing because distractor processing inhibits the channels leading to the same feature detector more strongly than channels leading to different feature detectors.

\section{Repetition Blindness}

Usually, the RLIE is investigated via experiments in which a single target is shown simultaneously with one or more distractors. In contrast, many other studies have used rapid serial visual presentation (RSVP). In an RSVP experiment, participants observe a sequence of stimuli presented in rapid succession at the same location. If participants have to report the stimulus sequence in the correct order, an interesting result is that repeated stimuli (i.e., the second instance from a pair of identical stimuli, e.g., $A B C \boldsymbol{B} E$ ) are less likely to be reported than unrepeated stimuli (e.g., $A B C \boldsymbol{D} E$ ). This phenomenon, called repetition blindness, has been found for different pairs of identical visual stimuli, including words (Kanwisher, 1987), letters (Kanwisher, 1991), digits (Bavelier \& Potter, 1992), and color patches (Kanwisher, 1991). Yet, for $\mathrm{RB}$ to occur, the stimulus onset asynchrony (SOA) between repeated stimuli must not exceed $500 \mathrm{msec}$ (Chun, 1997; Kanwisher, Kim, \& Wickens, 1996).

The most influential explanation for RB is the token individuation model (Epstein \& Kanwisher, 1999; Kanwisher, 1987; for alternative explanations, see Armstrong \& Mewhort, 1995; Fagot \& Pashler, 1995; Luo \& Caramazza, 1995, 1996). The token individuation model distinguishes between two forms of visual representations, types and tokens, and two processes operating on these representations, type activation and token individuation. A visual type is the representation of a stimulus category in long-term visual memory. A visual token is the representation of a specific instance of a stimulus in space and time. When a stimulus is presented, the corresponding type is activated automatically. However, before a stimulus becomes reportable, an activated type must be linked to an episodic token - that is, the token has to be individuated. According to the token individuation model, RB occurs because only one token can be linked to a type at a time.

Given the obvious similarity between the RLIE and $\mathrm{RB}$, the question arises whether these effects are produced by the same or by different mechanisms. Sometimes the RLIE has been presented as a case of RB without any further explanation (e.g., Park \& Kanwisher, 1994). Yet there are substantial methodological differences between the two paradigms. RB usually occurs when two identical visual stimuli are presented serially at the same location and participants must report both stimuli. Therefore, RB is attributed to a processing bottleneck that exclusively impairs identification of the repeated stimulus. In contrast, the RLIE usually occurs when two stimuli are presented at different locations and participants have to report only one of them. Therefore, the RLIE has been attributed to a deficit in parallel processing of identical stimuli that affects the processing of all stimuli equally (cf. Bjork \& Murray, 1977; Santee \& Egeth, 1980).

There have been demonstrations of RB with stimuli presented simultaneously at different locations (e.g., Kanwisher et al., 1996; Luo \& Caramazza, 1996). However, in these studies, participants were instructed to report the stimuli in a particular order (e.g., clockwise in the Luo and Caramazza, 1996, study), which most likely induced a corresponding order of processing. Moreover, several studies have tested whether RB occurs when participants have to report only one item (e.g., the last stimulus) from a series of stimuli. Under these conditions, both repetition priming (Kanwisher, 1987, Experiment 3) and RB (Kanwisher \& Potter, 1990, Experiment 6) have been observed. The reason for these discrepant results is unclear.

\section{Flanker Compatibility Effect and Redundancy Gain}

Charles Eriksen and his colleagues (e.g., B. A. Eriksen \& C. W. Eriksen, 1974) were also interested in the questions of whether and how adjacent distractors affect the identification of targets when the target appears at a known location in the visual field. In a typical Eriksen task, several letters are presented simultaneously. The target letter always appears in the central position. Two possible target letters are assigned to the left response key (e.g., $A$ and $B$ ), and two are assigned to the right response key (e.g., $C$ and $D$ ). Distractors (or "flankers") occupy the adjacent locations. In different conditions, distractors are identical to the target (e.g., $A A A$ ), response compatible (e.g., $B A B$ ), response incompatible (e.g., $C A$ $C$ ), or neutral (e.g., $E A E$; the letter $E$ is not assigned to one of the response keys). The main dependent variable is reaction time (RT) to the target.

When compared with a baseline condition in which the target appears alone, identical distractors do not seem to affect RTs to target stimuli. In contrast, compatible distractors seem to produce some interference (i.e., slower RTs), whereas incompatible distractors produce much interference (e.g., B. A. Eriksen \& C. W. Eriksen, 1974; C. W. Eriksen \& B. A. Eriksen, 1979; Miller, 1991). These effects decrease as the spatial distance between the target and the distractors increases (e.g., B. A. Eriksen \& C. W. Eriksen, 1974; Flowers \& Wilcox, 1982). Usually, the different conditions produce similar effects both in RTs and in error rates (accuracy). When compared with a different baseline condition in which neutral distractors accompany the target, identical and compatible distractors facilitate performance, whereas incompatible distractors impair performance (cf. Kinchla, 1992). The neutral-distractor condition appears to be a more adequate baseline condition than the target-alone condition because the former condition produces the same "filtering costs" (Kahneman, Treisman, \& Burkell, 1983) as the critical conditions. 
Usually the described pattern of results is explained by assuming limits in focusing spatial attention, in combination with effects of response priming and response competition. According to C. W. Eriksen and colleagues (e.g., C. W. Eriksen \& Schultz, 1979), participants focus their attentional resources upon the target position in advance. Yet because the focus of attention cannot be restricted to very small areas, irrelevant stimuli that are located close enough to the target will be processed in parallel with the target. The effects of different distractor types are explained in the following way (cf. Fournier \& Eriksen, 1990; Kinchla, 1992; Taylor, 1977): Incompatible distractors produce interference because they activate a conflicting response code (response competition). Compatible distractors produce facilitation because they activate the same response code as the target. Identical distractors (sometimes) produce more facilitation than compatible distractors because identical distractors activate both the same perceptual and response codes as the target. The facilitatory effect of identical targets is sometimes called redundancy gain (e.g., Miller, 1982). In the following discussion, we refer to both the facilitatory effects of identical distractors and the inhibitory effects of incompatible distractors as the flanker compatibility effect.

The FCE obviously conflicts with the RLIE (cf. C. W. Eriksen \& B. A. Eriksen, 1979). In particular, both effects have been explained on the basis of the assumption that distractors and target are processed in parallel. Yet parallel processing of identical targets produces interference in one case, whereas parallel processing of identical targets produces facilitation in the other. Three attempts have been made to resolve this contradiction. As a first attempt, Bjork and Murray (1977; Santee \& Egeth, 1982) proposed that RTs and accuracy might not measure the same aspects of visual (letter) recognition. In particular, the idea is that under data-limited conditions (i.e., masking), "accuracy frequently appears to be sensitive to early perceptual interference between target and noise items, whereas RT appears to be sensitive to later processes involved in response interference" (Santee \& Egeth, 1982, p. 499).

C. W. Eriksen and colleagues made a different attempt to solve this conflict. They suggested that the RLIE does not arise from perceptual interference, but from a response bias (C. W. Eriksen, Morris, Yeh, O'Hara, \& Durst, 1981). The authors argued that participants in the Bjork and Murray task might notice that different distractors accompany targets more often than do identical distractors. This observation might lead participants to report a target that is different from the distractor in those trials in which only the distractor had been identified. However, deficits in perceiving identical stimuli that are presented simultaneously have also been observed in tasks in which this "distractor-opposite bias" is very unlikely to occur (e.g., Mozer, 1989).

A third attempt to reconcile the RLIE and the FCE was made by Keren and Boer (1985). These authors focused on a further methodological difference between the Eriksen task and the Bjork and Murray task. In the former task, participants always know the target position in advance, which is not the case in the latter task. To investigate the role of positional knowledge, Keren and Boer presented two (identical or nonidentical) stimuli simultaneously under data-limited conditions and measured the accuracy of target identification. They gave participants advance information, however, about the target position. When target position was indicated by precues, no difference between identical and nonidentical distractors was observed (i.e., the RLIE had disappeared). Moreover, when target position was predictable, because the target always appeared at the same location, identical distractors produced facilitation, whereas incompatible distractors (i.e., the alternative target) produced interference. To explain their results, Keren and Boer suggested that participants can focus attentional resources on a predictable target position, which eliminates the effects of distractors on perceptual processing of the target. The problem with this account is that it does not explain the observation of redundancy gains.

\section{Two Conflicts and a Possible Solution}

The RLIE presents a challenge for researchers on visual perception. On one hand, the RLIE closely resembles RB, but the corresponding explanations are contradictory. The RLIE is explained on the assumption that two identical stimuli can be identified in parallel, giving rise to mutual (symmetrical) interactions between processing of each stimulus. In contrast, the token individuation model assumes that parallel identification of two identical stimuli is impossible (e.g., Epstein \& Kanwisher, 1999). Further discrepancies arise between the RLIE and the FCE. The RLIE has been claimed to represent inhibitory effects that occur when identical stimuli are processed in parallel (cf. Bjork \& Murray, 1977). In contrast, the FCE has been claimed to consist of both facilitation, when identical (and compatible) stimuli are processed in parallel, and interference, when incompatible stimuli are processed in parallel. How can these discrepancies be resolved?

We propose a solution that rests on the assumption that the RLIE arises - as does RB — when two identical stimuli are identified serially. In the Bjork and Murray task, participants do not know in advance which of two stimuli will be the target. Therefore, they identify both the distractor and the target, and we assume that identification proceeds serially in random order. This means that participants identify the distractor before the target in approximately one half of the trials, and they identify the target before the distractor in the other half. Furthermore, we assume that RB arises in the $50 \%$ of trials in which an identical distractor is identified before the target, but not when the target is identified before an identical distractor.

Our account makes several predictions that are tested in three experiments. The first prediction is that considerably more RB should result for situations in which the 
distractor is always identified before the target. This hypothesis is tested in Experiment 1. The second prediction is that a predictable target location should eliminate the RLIE (RB) with simultaneously presented distractors and produce an FCE in identification accuracy instead (cf. Keren \& Boer, 1985). However, a predictable target location might not eliminate RB from identical distractors that precede the target, because the distractor might automatically grab attention and hence affect target processing. The latter predictions are tested in Experiments 2 and 3 .

Finally, we would like to stress two implications of our account for the RLIE. First, our assumption is that the RLIE does not arise from a response bias, as was suggested by C. W. Eriksen and colleagues, but rather from interference between overlapping processing of two stimuli. This means that the RLIE should still occur when the distractor-opposite bias described above is unlikely to occur. This was the case in Experiments 1 and 2. Moreover, these experiments explicitly tested for possible response biases. Second, identification accuracy for masked stimuli should be equally sensitive to perceptual interference and response competition-a prediction that contrasts with previous claims (e.g., Santee \& Egeth, 1982).

\section{EXPERIMENT 1}

This experiment had two goals. The first was to test our interpretation of the RLIE. According to this account, the RLIE occurs with concurrent presentation of two identical stimuli at unpredictable target locations, because participants identify target and distractor serially and in random order. As a result, an identical distractor is assumed to produce RB (= RLIE) for the target in the $50 \%$ of all trials with identical distractors in which the distractor is identified before the target. In the remaining half of the trials, though, the distractor should not impair target identification. If this idea is correct, RB (i.e., RLIE) with simultaneous presentation should be significantly less than RB in a situation in which the distractor is presented before the target. In the latter case, a single distractor should almost always be identified successfully before the actual target appears, and RB should arise in almost every trial with identical distractors.

In each trial of Experiment 1, two stimuli were presented at different locations on a computer screen. The two stimuli were either identical or different (i.e., incompatible). A mask that followed one of the stimuli served as a postcue indicating the target position. Importantly, as in Bjork and Murray's (1977) study, the target position was not predictable. Besides varying the type of distractor, the order of presenting the target and the distractor was also varied. The distractor appeared either $280 \mathrm{msec}$ before, simultaneously with, or $280 \mathrm{msec}$ after the target. In the distractor-before-target condition, we expected large RB effects - that is, more identification errors (i.e., confusion errors and misses; see below) - with identical in comparison with nonidentical distractors. Because the first stimulus might be the target, it should always be identified, and identical distractors should almost always produce RB for subsequently presented targets. The simultaneous-presentation condition is equivalent to Bjork and Murray's task. In this case, we also expected RB (i.e., RLIE), but this effect should be considerably smaller than in the distractor-before-target condition because, in the simultaneous-presentation condition, RB should only result for the $50 \%$ of trials with identical distractors in which the distractor is identified before the target. Finally, in the distractor-after-target condition, we expected no difference in the effects of identical and nonidentical distractors.

The second goal of Experiment 1 was to investigate the role of response bias for the RLIE. According to C. W. Eriksen et al. (1981), the RLIE originates from a distractor-opposite bias. Participants may be inclined to report the alternative target more frequently when they have actually only identified the distractor. In Experiment 1 , we tried to prevent response bias in two ways: First, distractors were equally often identical and nonidentical to the target. Second, besides reporting one of the two targets, participants could also report that they have not seen a stimulus. Both precautions should prevent the occurrence of a response bias. Yet because such a bias might have already existed before our experiment, we tried to measure the bias directly. Therefore, in one third of the trials, a distractor but no target was presented. The correct response was to report "no target presented," but false alarms were possible. If participants' judgments are related to the identity of the distractor in perceptually unclear situations, then this bias should show up in false alarm rates. A higher percentage of distractor-incongruent than of distractor-congruent false alarms, for example, might indicate the presence of a distractor-opposite bias.

\section{Method}

Participants. Twenty volunteers (11 female) participated in the experiment. They ranged from 15 to 46 years in age (mean age $=$ 26). All participants of the present and the subsequent experiments reported to have normal or corrected-to-normal vision and were paid $€ 6.15$ for participation in the experiment.

Apparatus and Stimuli. The experiments were run on an Apple Macintosh computer with MacProbe. Visual stimuli were presented on a 17-in. monitor with a screen refresh rate of $75 \mathrm{~Hz}$ and a luminance of $40 \mathrm{~cd} / \mathrm{m}^{2}$. Perceptual judgments were collected with the mouse. The experiment was carried out in a dimly lit and soundproof chamber.

The visual stimuli can be described as small diamonds with limbs at the top and bottom (see Figure 1). The main axis of one stimulus was oriented from the lower left to the upper right, whereas the main axis of the second stimulus was oriented from the upper left to the lower right. From a viewing distance of $50 \mathrm{~cm}$, these stimuli subtended about $0.8^{\circ}$ of visual angle horizontally and $1.6^{\circ}$ vertically. The mask consisted of randomly arranged lines having the same diagonal orientation as the components of the stimuli and subtended $1.0^{\circ} \times 2.0^{\circ}$ of visual angle. All visual stimuli were displayed in black on a white background. Stimuli were presented $1.6^{\circ}$ above and $1.6^{\circ}$ below the fixation point. 
A

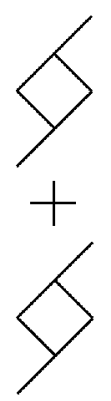

B

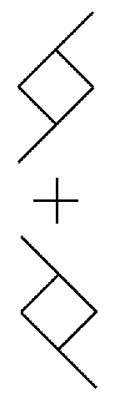

C

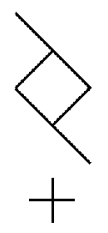

$\mathrm{D}$

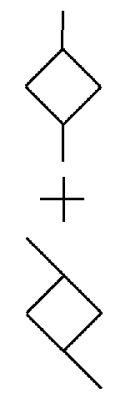

Figure 1. Examples for experimental conditions, in which the distractor appears above and the target below fixation (A) with an identical distractor, (B) with a nonidentical (incompatible) distractor, (C) with only a distractor but no target, and (D) with a neutral distractor (which was used only in Experiment 3).

Procedure. An experimental trial started with the presentation of the fixation cross at the screen center; the cross remained on the screen until the end of the trial. In the distractor-before-target condition, the distractor appeared $1 \mathrm{sec}$ after the onset of the fixation cross, either above or below fixation. The target appeared $280 \mathrm{msec}$ later at the opposite location. In the simultaneous-presentation condition, both the distractor and the target appeared $1 \mathrm{sec}$ after the onset of the fixation cross, one stimulus above and the other below the fixation cross. In the distractor-after-target condition, the target appeared $1 \mathrm{sec}$ after the onset of the fixation point, either above or below fixation, and the distractor appeared $280 \mathrm{msec}$ later at the opposite location. In each condition, the target stimulus appeared for an individually adjusted presentation time (see below) and was then replaced by a mask, which remained for $1 \mathrm{sec}$. The distractor and the mask disappeared simultaneously. In one third of the trials of each condition, however, no target was presented. In these cases, the mask was presented alone.

Half a second after the offset of mask and distractor, the judgment screen appeared. The judgment screen showed the two possible targets and a " 0 " on the horizontal meridian of the screen. These stimuli changed their relative positions randomly from trial to trial. The participants had to report the identity of the masked stimulus in the last trial by clicking on the corresponding symbol with the mouse. For each of the two possible target stimuli, the corresponding symbol had to be chosen, whereas the " 0 " had to be chosen if no target stimulus had been detected. The instruction stressed the fact that there was no systematic relationship between the unmasked distractor and the masked target, and the participants were told to guess if uncertain about the identity of the target.

When no error had occurred, the next trial started with a delay of $1 \mathrm{sec}$ after a response. If participants had reported the wrong stimulus, a corresponding error message occurred for one additional second. There were three possible errors and, hence, three corresponding messages. If a target had been presented and the participant reported the alternative stimulus, this error was termed a confusion error, and the message was "You chose the wrong stimulus!" If a target had been presented and the participant reported the " 0 ," this error was termed a miss, and the message was "You missed a stimulus!" Finally, if no target had been presented and the participant reported one of the stimuli, this error was termed a false alarm, and the message was "No stimulus presented!" After each block, the participants were informed about the percentage of error-free trials.

To avoid ceiling or floor effects, the presentation time for the target stimuli was adjusted to achieve $75 \%$ performance accuracy, sep- arately for each SOA condition. The following staircase procedure was used: After each experimental block, the presentation time was decreased by one screen refresh $(13 \mathrm{msec})$ if the percentage of correctly identified targets was above $90 \%$. It was increased by one refresh if performance was below $60 \%$.

The experimental phase contained 13 blocks of 36 trials ( 2 distractors $\times 3$ targets $\times 3$ SOAs $\times 2$ repetitions), yielding a total of 468 trials. The experimental phase was preceded by a practice phase consisting of 5 blocks of 18 trials. In the first block of the practice phase, the target stimuli were always presented for $67 \mathrm{msec}$ (five screen refreshes). During the following practice blocks, the presentation duration was adjusted according to the staircase procedure. The presentation durations for the three SOA conditions from the last practice block were fed into the first experimental block. The participants could take a rest whenever a block had ended. The whole experiment took about 1 hour.

Design. Two independent variables were crossed in a $3 \times 3$ withinsubjects design. The first factor was the relationship between the distractor and the target, with the levels identical, nonidentical, and none (when a distractor but no target was presented). The second factor was the order of presenting the distractor and the target (or mask, when no target was presented), with the levels distractor before target, simultaneous presentation, and distractor after target. However, we planned to separately analyze the error rates for trials with and without targets. In trials containing targets, two types of errors - confusion errors and misses - could occur. These two error types were analyzed as a function of the target-distractor relationship and the presentation order. In trials without targets, only false alarms could occur. In order to test for possible response biases, false alarm rates were analyzed as a function of distractor congruency (distractor-congruent vs. distractor-incongruent) and of presentation order (mask first vs. distractor first).

\section{Results}

The mean adjusted presentation times for the target were $62 \mathrm{msec}(S D=25 \mathrm{msec}), 76 \mathrm{msec}(S D=23 \mathrm{msec})$, and $53 \mathrm{msec}(S D=25 \mathrm{msec})$ for the distractor-beforetarget, simultaneous-presentation, and distractor-aftertarget conditions, respectively. A one-way analysis of variance (ANOVA) revealed significant differences among the presentation times $\left[F(2,38)=24.83, M S_{\mathrm{e}}=109.42\right.$, $p<.001]$. Thus, in order to achieve approximately equal levels of identification performance, participants needed different amounts of time for processing targets presented after, simultaneously with, and before the distractor.

Target-present trials. In the target-present trials, participants could make two types of errors: A confusion error was to report the alternative stimulus instead of the stimulus that had actually been presented as a target. A miss was to report the absence of an actually presented target. Figure 2 shows the observed percentages of confusion errors and misses. The percentages of misses and confusion errors were entered into separate $2 \times 3$ ANOVAs, with target-distractor relationship (identical vs. nonidentical) and presentation order as within-subjects factors.

Overall, misses were quite rare $(M=4.4 \%)$. The ANOVA of misses only revealed a significant main effect of presentation order $\left[F(2,38)=8.64, M S_{\mathrm{e}}=34.84\right.$, $p<.01]$. This main effect indicates a decrease of misses $(1.3 \%)$ with simultaneous presentation in comparison with the distractor-before-target $(6.2 \%)$ and the distractorafter-target $(5.8 \%)$ conditions, respectively. The main effect of target-distractor relationship $[F(1,19)=1.61$, 


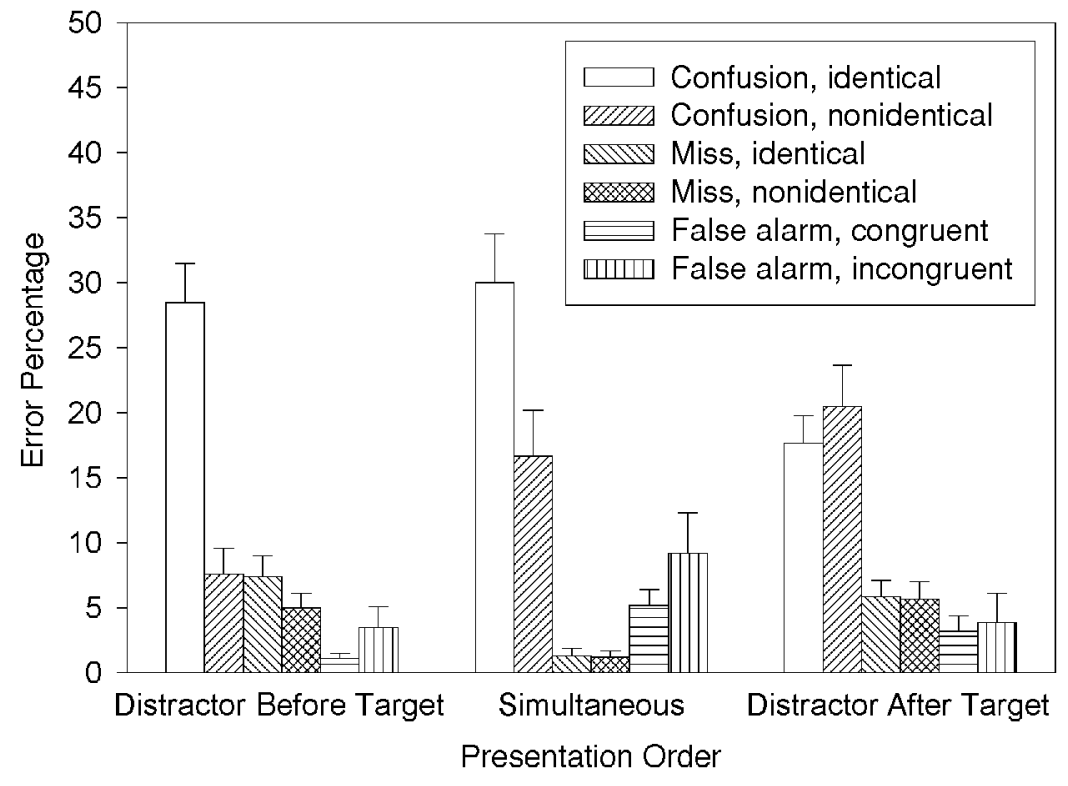

Figure 2. Results of Experiment $1(N=20)$. Percentages of confusion errors, misses, and false alarms in identification performance, as a function of distractor type (identical or nonidentical) and presentation order. Error bars represent standard errors between participants.

$p=.22]$ and the two-way interaction $[F(2,38)=1.95$, $p=.16]$ were not significant.

Confusion errors $(20.2 \%)$ were much more frequent than misses. The ANOVA of confusion errors revealed significant main effects of presentation order $[F(2,38)=$ $\left.5.74, M S_{\mathrm{e}}=55.60, p<.01\right]$ and target-distractor relationship $\left[F(1,19)=5.21, M S_{\mathrm{e}}=628.64, p<.05\right]$, as well as a significant two-way interaction $[F(2,38)=19.62$, $\left.M S_{\mathrm{e}}=74.35, p<.001\right]$. The main effect of presentation order indicates an increase of confusion errors (23.4\%) with simultaneous presentation in comparison with the distractor-before-target $(18.0 \%)$ and the distractor-aftertarget $(19.1 \%)$ conditions, respectively. The main effect of target-distractor relationship reflects the presence of more confusion errors with identical distractors (25.4\%) than with nonidentical distractors (14.9\%). Yet, as indicated by the significant interaction, this effect was in turn strongly affected by presentation order. In fact, identical distractors strongly impaired performance when they preceded the target, increasing confusion errors by $21 \%[t(19)=4.83, p<.001]$. With simultaneous presentation, identical distractors still impaired performance, increasing confusion errors by $13 \%[t(19)=2.06, p=$ $.05]$. In contrast, identical distractors produced a nonsignificant benefit (3\%) when they followed the target $[t(19)=0.67, p=.51]$.

Finally, we tested whether the perceptual impairments produced by identical distractors were different for the three order conditions. In fact, the impairment for the distractor-before-target condition (21\%) was larger than that for the simultaneous-presentation condition (13\%) $[t(19)=2.08, p=.05$ (two-tailed) $]$. Moreover, the latter effect was significantly different from the $3 \%$ difference for the distractor-after-target condition $[t(19)=3.71$, $p<.01]$.

Target-absent trials. False alarms were the only possible errors in target-absent trials. Importantly, false alarms could be congruent or incongruent to the actually presented distractor and, therefore, reveal some clues about a response bias that participants might apply in perceptually unclear situations. Figure 2 shows the observed percentages of distractor-congruent and -incongruent false alarms as a function of presentation order.

False alarms $(M=4.3 \%)$ were nearly as frequent as misses $(M=4.4 \%)$. This makes sense, because both types of errors reflect participants' ability to discriminate target-present from target-absent trials. False alarms were subjected to an ANOVA, with distractor congruency and presentation order as within-subjects factors. The main effect of presentation order was significant $\left[F(2,38)=6.81, M S_{\mathrm{e}}=38.98, p<.01\right]$. This main effect indicates an increase of false alarms $(7.2 \%)$ with simultaneous presentation in comparison with the distractorbefore-target $(2.3 \%)$ and the distractor-after-target $(3.5 \%)$ conditions, respectively. Importantly, neither the main effect of distractor congruency $\left[F(1,19)=1.98, M S_{\mathrm{e}}=\right.$ $85.46, p=.18]$ nor the two-way interaction $[F(2,38)=$ $\left.1.97, M S_{\mathrm{e}}=14.41, p=.15\right]$ was significant.

\section{Discussion}

Experiment 1 had two goals. The first was to test the hypothesis that the RLIE arises from serial identification when two stimuli are presented simultaneously and target position is not predictable. In particular, we assumed 
that stimuli are identified in random order and that RB (i.e., RLIE) occurs in the $50 \%$ of trials with identical distractors in which the distractor is identified before the target. In the remaining $50 \%$ of the trials, in which the target is processed before the distractor, identical distractors should not impair target processing. To test this prediction, the effects of simultaneously presented distractors were compared with the effects of distractors that preceded the target. For the latter situation, we assumed that the distractor should always be identified before the target, and hence an identical distractor should almost always impair target identification.

The results of Experiment 1 confirm these predictions. We found significantly larger RB in the distractor-beforetarget condition than in the simultaneous-presentation condition. This finding suggests that the RLIE observed by Bjork and Murray (1977) is a case of RB that arises in about half of all trials with identical distractors, when the distractor is identified before the target. Also in accordance with the predictions, no effects were observed in the distractor-after-target condition.

The second goal of Experiment 1 was to investigate the role of response biases in the RLIE. According to C. W. Eriksen et al. (1981), the RLIE might result from participants' preference for reporting the alternative stimulus when only the distractor has been identified (distractor-opposite bias). To prevent our task from inducing this bias, identical and nonidentical distractors were equally frequent. Moreover, participants were not forced to report a target, but they could also report "no stimulus presented/perceived." Finally, we also analyzed whether false alarm rates, obtained only from distractoronly trials, were either congruent or incongruent with the distractor. Neither was the case. Therefore, we conclude that perceptual interference (RB) between identical stimuli, and not response bias, produced the effects observed in Experiment 1 (cf. Kanwisher et al., 1996). The next experiment investigated how knowledge of target position changes the effects of identical distractors.

\section{EXPERIMENT 2}

Experiment 2 was identical to Experiment 1, except that distractor and target appeared at predictable locations in Experiment 2. For one half of the participants, the distractor always appeared above, and the target always below, fixation, whereas the reverse was true for the remaining participants. According to our account, an important precondition for the RLIE is that participants cannot predict the target position. When the target position is predictable, however, participants can focus attention upon the target position in advance. Moreover, when the target and the distractor appear simultaneously, focused attention will facilitate target identification, but distractors might be processed in parallel with the target if they are located close enough to it. When parallel processing takes place, however, identical distractors might facilitate and incompatible distractors inhibit target pro- cessing. As a result, for the simultaneous-presentation condition of Experiment 2, we expected better performance (i.e., fewer errors) with identical than with nonidentical distractors.

The situation is different when the distractor precedes the target. In this case, knowing the target position might not prevent the distractor from being identified, because the appearance of a single distractor in an empty display might automatically grab attention (cf. Yantis, 1993), which might facilitate distractor identification. Therefore, a single distractor that precedes the target might cause RB, even though participants know that this stimulus is a distractor. As in Experiment 1, we analyzed false alarm rates as a function of distractor congruency in order to detect possible response biases. Finally, in accord with the results of Experiment 1, no effects were expected for the distractor-after-target condition.

\section{Method}

Participants. Twenty-four new participants (16 female) volunteered in the experiment. They ranged from 20 to 32 years in age (mean age $=23$ ).

Apparatus and Stimuli. Apparatus and stimuli were the same as in Experiment 1.

Procedure. Experiment 2 differed from Experiment 1 only in that both the target and the distractor appeared at constant (i.e., predictable) screen locations. For half of the participants, the target always appeared above fixation, whereas the distractor always appeared below fixation. For the other half, the opposite was true.

Design. The design of Experiment 2 was identical to that of Experiment 1 .

\section{Results}

The mean presentation times for the target were $35 \mathrm{msec}$ $(S D=12 \mathrm{msec}), 32 \mathrm{msec}(S D=9 \mathrm{msec})$, and $27 \mathrm{msec}$ $(S D=9 \mathrm{msec})$ for the distractor-before-target, simultaneous-presentation, and distractor-after-target conditions, respectively. A one-way ANOVA indicated significant differences between these conditions $[F(2,46)=$ $\left.33.78, M S_{\mathrm{e}}=11.78, p<.001\right]$. Thus, in order to achieve approximately equal levels of identification performance, participants needed different amounts of time for processing targets presented after, simultaneously with, and before the distractor.

Target-present trials. Figure 3 shows the observed percentages of confusion errors and misses as a function of the two experimental factors. The percentages of confusion errors and misses were entered into separate $2 \times$ 3 ANOVAs with target-distractor relationship and presentation order as within-subjects factors. The ANOVA of misses revealed no significant effects $(F<1$ for both main effects; $F=1.43, p=.25$, for the interaction).

Again, confusion errors (15.7\%) were more frequent than misses (7.7\%). An ANOVA of confusion errors revealed no effect of presentation order $(F<1)$, but the main effect of target-distractor relationship $[F(1,23)=$ $\left.10.75, M S_{\mathrm{e}}=105.39, p<.01\right]$ and the two-way interaction $\left[F(2,46)=19.26, M S_{\mathrm{e}}=78.20, p<.001\right]$ were significant. The main effect of target-distractor relationship reflects the presence of more confusion errors 


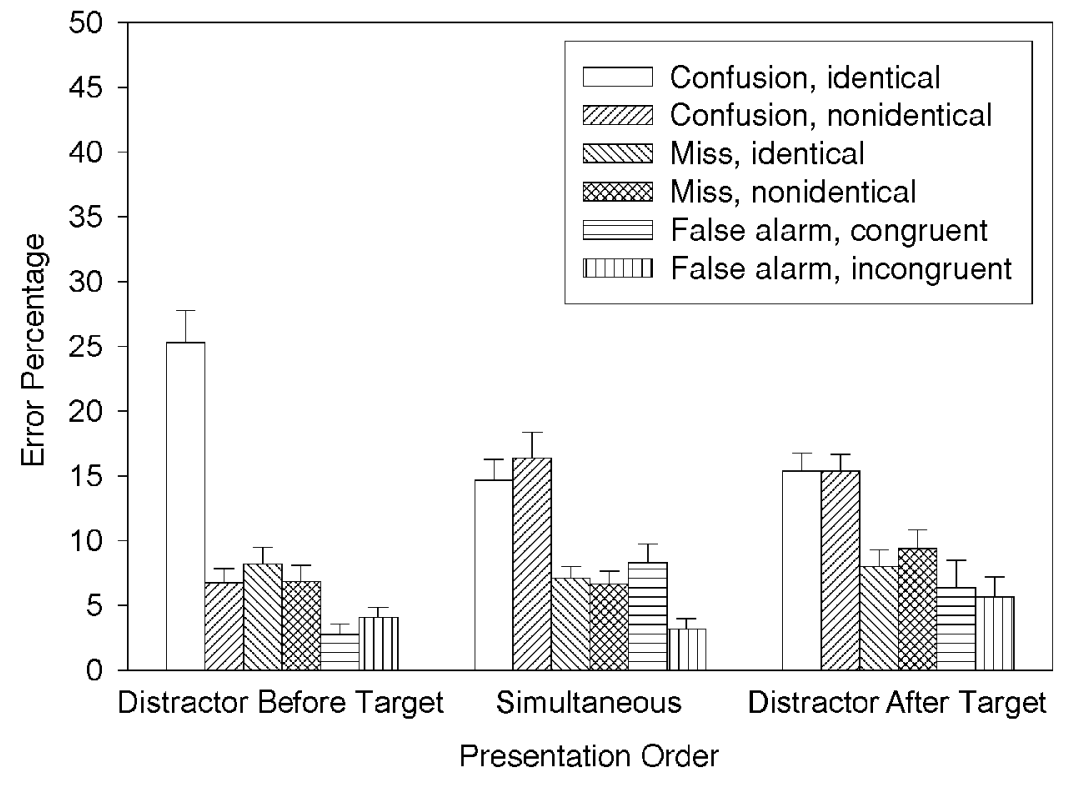

Figure 3. Results of Experiment $2(N=24)$. Percentages of confusion errors, misses, and false alarms in identification performance, as a function of distractor type (identical or nonidentical) and presentation order. Error bars represent standard errors between participants.

with identical (18.5\%) than with nonidentical (12.9\%) distractors. This effect was strongly modulated by presentation order, as is indicated by the significant interaction. In fact, identical distractors strongly impaired performance when they preceded the target, increasing confusion errors by $18 \%[t(23)=5.91, p<.001]$. With simultaneous presentation, identical distractors slightly improved performance, decreasing confusion errors by a nonsignificant amount of $2 \%[t(23)=0.55, p=.59]$. Finally, when distractors followed the target, performance with identical and nonidentical distractors was indistinguishable $[t(23)=0.01, p=.99]$.

Target-absent trials. Figure 3 also shows the observed percentages of distractor-congruent and -incongruent false alarms as a function of presentation order (between distractor and mask). Again, false alarms (5.1\%) were approximately as frequent as misses $(7.7 \%)$. False alarms were subjected to an ANOVA, with distractor congruency and presentation order as within-subjects factors. Presentation order had no significant main effect $[F(2,46)=$ $\left.2.20, M S_{\mathrm{e}}=44.00, p=.12\right]$, but the main effect of distractor congruency $\left[F(1,23)=4.29, M S_{\mathrm{e}}=18.80, p=\right.$ $.05]$ and the two-way interaction $\left[F(2,46)=8.38, M S_{\mathrm{e}}=\right.$ $14.99, p<.01]$ were significant. The significant main effect indicates that distractor-congruent false alarms (5.8\%) were more frequent than distractor-incongruent false alarms $(4.3 \%)$. This effect was only present, though, with simultaneous presentation of distractors and targets. In this case, distractor-congruent false alarms $(8.3 \%)$ were significantly more frequent than distractor-incongruent false alarms $(3.2 \%)[t(23)=5.05, p<.01]$. In contrast, the percentages of distractor-congruent and -incongruent false alarms, respectively, were not different in the two remaining presentation orders [for both, $t(23)<1.30$, $p>.20]$.

\section{Discussion}

Experiment 2 investigated how predictability of the target and distractor locations might affect the pattern of results that was observed in Experiment 1 for unpredictable locations. As expected, when the distractor preceded the target, we still observed RB in Experiment 2. Thus, even when participants know that a single stimulus is a distractor, the sudden onset of this stimulus grabs attention and facilitates its identification. An identified distractor causes RB for subsequently presented identical targets.

For simultaneous presentation, predictions of the present experiment differed from those of Experiment 1. In Experiment 2, participants could focus attention upon the target location in advance, which should have facilitated the identification of a target that appeared simultaneously with a distractor. Yet the distractor might still have been processed in parallel with the target, leading to better performance with identical than with nonidentical distractors. In fact, we observed fewer errors with identical than with nonidentical distractors in the simultaneouspresentation condition, but this difference was not statistically significant. A possible reason for the absence of a significant effect is the relatively large spatial distance between target and distractor locations $\left(\sim 3.2^{\circ}\right)$. Previous studies have shown that the FCE decreases with increasing spatial separation between target and distractor stimuli (e.g., B. A. Eriksen \& C. W. Eriksen, 1974; Miller, 
1991). Therefore, significant effects might also occur in our task when the spatial separation between target and distractor is reduced, as was done in Experiment 3.

As in Experiment 1, our analyses of false alarm rates revealed no evidence for a distractor-opposite bias that might explain inferior performance with identical distractors. Surprisingly, however, participants produced more distractor-congruent than -incongruent false alarms for the simultaneous-presentation condition of Experiment 2. So far, we do not have an explanation for this effect. In sum, however, the results of the false alarm analyses support the conclusion that the negative effects of identical distractors are caused by perceptual interference and not by response strategies. The next experiment is basically a replication of Experiment 2 in which we reduced spatial target-distractor separation and added two neutral conditions.

\section{EXPERIMENT 3}

Experiment 3 replicated Experiment 2 with a smaller target-distractor distance $\left(\sim 1.6^{\circ}\right)$. This change should provoke better performance with identical than with nonidentical distractors in the simultaneous-presentation condition. Moreover, to be able to distinguish between facilitatory and inhibitory effects of the distractors, two baseline conditions were added. In one, the target was presented without a distractor. In the other, the target was presented with a neutral distractor that never appeared as a target. The distractor appeared either $280 \mathrm{msec}$ before or simultaneously with the target. The distractor and target positions were constant - that is, predictable. We expected best performance in the baseline condition without distractor, because distractor stimuli are likely to produce filtering costs in target processing (Kahneman et al., 1983). When the distractor preceded the target, we expected RB - that is, more errors with identical than with neutral and incompatible (i.e., alternative target) distractors. The latter two conditions should not differ (cf. Bjork \& Murray, 1977). With simultaneous presentation, we predicted superior performance with identical as compared with neutral distractors (i.e., a redundancy gain) and inferior performance with incompatible as compared with neutral distractors (i.e., response competition).

\section{Method}

Participants. Twenty-two new participants (17 female) volunteered in the experiment. They ranged from 17 to 42 years in age (mean age $=25)$.

Apparatus and Stimuli. The same apparatus and stimuli were used as in the preceding experiments. Moreover, a third stimulus was introduced as a neutral distractor - that is, this stimulus never occurred as a target (see Figure 1D). Stimuli were presented $0.8^{\circ}$ above and $0.8^{\circ}$ below the fixation point.

Procedure. The procedure of Experiment 3 was the same as that in Experiment 2, except for the following changes: First, a target was presented in every trial. As a result, only confusion errors and misses, but not false alarms, were possible. Second, there were four target-distractor relationships: identical (distractor was same as target), incompatible (alternative target), neutral (distractor was never a target), and none (target presented alone). Third, there were only two orders of presenting the target and the distractor: Either the presentation of the distractor started $280 \mathrm{msec}$ before the target or distractor and target were presented simultaneously. Fourth, the distractor was presented for the same brief period of time as the target. The first and the third changes were made in order to compensate for the increase in the number of trials caused by the introduction of two additional target-distractor relationship conditions. The fourth change was not expected to alter the qualitative pattern of results. If reducing the presentation duration of the distractor makes a difference at all, it should decrease the distractor's impact on target processing.

Note that the judgment screen was the same as in the preceding experiments. That is, the participants still had to choose between three alternative judgments (Target 1 , Target 2, no target perceived). The no target perceived response was included because we did not want to force the participants to choose one of the targets when they had not identified anything. The other details of the procedure of Experiment 3 were identical to those of Experiment 2.

The experimental phase contained 10 blocks of 32 trials ( 4 distractors $\times 2$ targets $\times 2$ orders $\times 2$ repetitions), yielding a total of 320 trials. The experimental phase was preceded by a practice phase consisting of 5 blocks of 16 trials. The whole experiment lasted 1 hour.

Design. Two independent variables were crossed in a $4 \times 2$ within-subjects design. The first factor was distractor type (identical, incompatible, neutral, or none), and the second was presentation order (distractor before target or simultaneous presentation). Dependent variables were the percentages of misses and confusion errors.

\section{Results}

Mean presentation times for the target were $39 \mathrm{msec}$ $(S D=26 \mathrm{msec})$ and $36 \mathrm{msec}(S D=25 \mathrm{msec})$ for the distractor-before-target and simultaneous-presentation conditions, respectively. These two means were not significantly different $[t(23)=0.20, p=.84]$. As in Experiment 2, presentation times for the distractor-beforetarget and simultaneous-presentation conditions were very similar, which is not surprising, because only one stimulus (i.e., the target at the known location) had to be identified in both conditions. In contrast, presentation time had been higher for the simultaneous condition than for the distractor-before-target condition in Experiment 1 because both stimuli had to be identified (since target location was not known in advance), and this process was more difficult with simultaneous presentation.

Target-present trials. Percentages of misses and confusion errors were entered into separate $4 \times 2$ ANOVAs, with distractor type and presentation order as withinsubjects factors. Overall, misses were very rare $(M=$ $1.4 \%$ ). This is not surprising, because participants knew that a target was always presented. The ANOVA of misses revealed no significant effects (all $F \mathrm{~s}<1$ ).

Confusion errors $(22.0 \%)$ were much more frequent than misses. Figure 4 shows the observed percentages of confusion errors as a function of the two experimental factors. An ANOVA revealed no effect of presentation order $[F(1,21)=1.18, p=.289]$, but the main effect of distractor type $\left[F(3,63)=18.27, M S_{\mathrm{e}}=77.30, p<\right.$ $.001]$ and the interaction $\left[F(3,63)=17.36, M S_{\mathrm{e}}=88.52\right.$, $p<.001]$ were significant. The main effect of distractor 


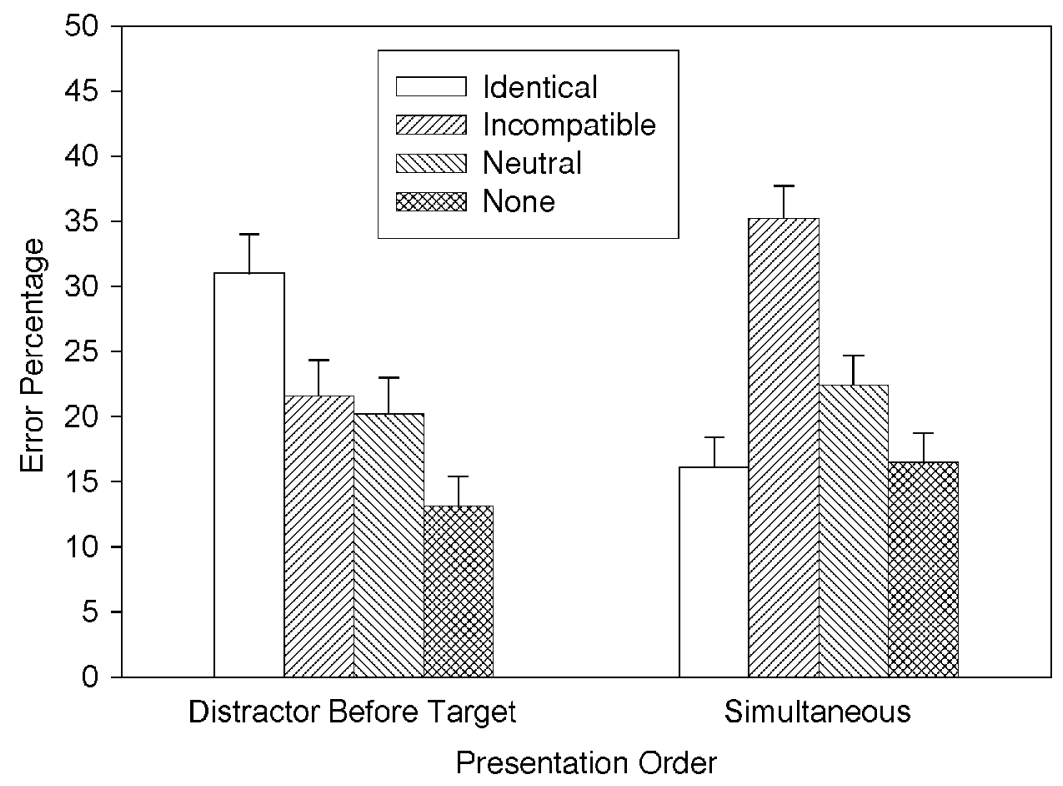

Figure 4. Results of Experiment $3(N=22)$. Percentages of confusion errors in identification performance, as a function of distractor type (identical, incompatible, neutral, or none) and presentation order. Error bars represent standard errors between participants.

type indicates that, independent of presentation order, performance was best without a distractor $(14.8 \%$ errors). A neutral distractor ( $21.3 \%$ errors) and an identical distractor (23.6\% errors) produced intermediate performance, whereas an incompatible distractor $(28.4 \%$ errors) produced the worst performance.

However, as indicated by the interaction, the effect of distractor type was strongly affected by presentation order. In fact, when the distractor preceded the target, identical distractors produced the worst performance (31.0\% errors), incompatible distractors ( $21.6 \%$ errors) and neutral distractors $(20.2 \%$ errors) produced intermediate performance, and no distractor produced the best performance ( $13.1 \%$ errors). In particular, identical distractors produced significantly more errors than were found in the incompatible-distractor $[t(21)=2.79, p<$ $.05]$, neutral-distractor $[t(21)=3.19, p<.01]$, and nodistractor $[t(21)=5.29, p<.001]$ conditions. In contrast, effects of incompatible distractors were not different from those of neutral distractors $[t(21)=0.61, p=$ $.55]$, but the former condition did produce more errors than the no-distractor condition $[t(21)=4.28, p<.001]$.

A different picture emerged when the distractor and the target were presented simultaneously. Here an identical distractor (16.1\% errors) allowed for performance as good as that found with no distractor $(16.5 \%)$. Neutral distractors (22.4\% errors) produced intermediate performance, and incompatible distractors $(35.2 \%)$ produced the worst performance. In particular, identical distractors produced as many errors as were found in the nodistractor condition $[t(21)=0.18, p=.86]$ and fewer errors than in either the neutral-distractor $[t(21)=2.50$, $p<.05]$ or incompatible-distractor $[t(21)=5.52, p<$ .001] conditions. In contrast, incompatible distractors produced more errors than were found in either the nodistractor $[t(21)=4.78, p<.001]$ or neutral-distractor $[t(21)=5.91, p<.001]$ condition.

\section{Discussion}

Experiment 3 compared the effects of a preceding distractor with those of a simultaneously presented distractor on the processing of a target at a predictable location. When the distractor preceded the target, only RB occurred - that is, more errors with identical than with either neutral or incompatible distractors. This result replicates those of Experiments 1 and 2 and shows that participants can hardly ignore a single stimulus, even when they know that this stimulus is a distractor. Moreover, the similarity between the results of Experiments 2 and 3 for the distractorbefore-target condition suggests that the duration of distractor presentation does not affect the pattern of results.

Yet for simultaneous presentation, the present experiment produced different results than did Experiment 2. In the present experiment, identical distractors facilitated processing of the target - that is, they elicited fewer errors than neutral distractors. Whether this redundancy gain is due to perceptual identity or response compatibility cannot be decided, however. Interestingly, performance with identical distractors was as good as that with targets presented alone. This result suggests that facilitation with identical distractors can outweigh the filtering costs imposed by the presentation of a distractor (cf. Fournier \& Eriksen, 1990). In contrast with identical distractors, incompatible distractors (i.e., the alternative 
target) interfered with target processing - that is, they elicited more errors than did neutral distractors. In other words, the simultaneous-presentation condition in Experiment 3 produced a typical FCE in identification accuracy for masked targets (cf. Keren \& Boer, 1985). The absence of similar effects in Experiment 2 can thus be attributed to the larger target-distractor distance.

\section{GENERAL DISCUSSION}

The major purpose of the present study was to identify the experimental conditions under which identical distractors facilitate or impair the identification of target stimuli, which should help clarify some existing discrepancies between several empirical phenomena and their explanations. The first discrepancy was identified between the RLIE, which has been attributed to a deficit in parallel encoding of identical stimuli (Bjork \& Murray, 1977), and RB, which has been attributed to a deficit in serial encoding of identical stimuli (Kanwisher, 1987). The relationship between these effects was investigated in Experiment 1, in which participants could not predict the locations of a to-be-reported target stimulus and a not-to-be-reported distractor stimulus. In this experiment, identical distractors produced significantly greater impairments in target processing when the distractor preceded the target than when it appeared simultaneously with the target. In other words, RB was significantly larger than the RLIE. This result suggests that not only RB but also the RLIE results from serial identification of a distractor before the target, which should occur in about half of all trials with simultaneous presentation of distractor and target. On the basis of this result, we conclude that the RLIE is an instance of RB that can be attributed to a deficit in identifying identical stimuli in a brief period of time.

A second conflict is that between the RLIE and the FCE. The FCE denotes the observation that identical distractors facilitate target processing (redundancy gain), and incompatible distractors interfere with it, when the distractor and the target occur simultaneously (e.g., Fournier \& Eriksen, 1990). On the surface, this pattern of results seems incompatible with the RLIE. Three proposals have been made in order to resolve the discrepancy: The first was that it might relate to the use of different dependent variables. In particular, the claim has been that accuracy might be more sensitive to interference at early stages of visual processing, whereas RT might be more sensitive to interference at later (response-related) stages of processing. This proposal is clearly inconsistent with the results of the present Experiment 3 and with those of Keren and Boer (1985). Both studies have shown that identical stimuli can improve identification accuracy and that identification accuracy is sensitive to interference between incompatible stimuli, which is assumed to arise at later stages of visual processing. Thus, different dependent variables do not seem to be the solution.

The second proposal to resolve the discrepancy between the RLIE and the FCE was to attribute the RLIE to re- sponse bias. According to C. W. Eriksen et al. (1981), the task used by Bjork and Murray (1977) and by Santee and Egeth (1980) tempts participants to report the alternative stimulus when the distractor but not the target can be identified. To prevent such a distractor-opposite bias, we presented identical and nonidentical distractors equally frequently in Experiments 1 and 2. Moreover, participants had the possibility to report "no stimulus presented/ perceived." Nevertheless, we observed both RB for serially presented stimuli and the RLIE for simultaneously presented stimuli. In addition, we analyzed whether false alarms, which occurred in trials without target presentation, were congruent or incongruent to the distractor, and we found no evidence for a distractor-opposite bias. In sum, our results support the conclusion that neither RB nor the RLIE is produced by response strategies (cf. Anderson \& Neill, 2002; Neill, Neely, Hutchison, Kahan, \& VerWys, 2002). Rather, we attribute these effects to deficits in perceptual encoding of identical stimuli (see Anderson \& Neill, 2002; Johnston, Hochhaus, \& Ruthruff, 2002; and Neill et al., 2002, for similar conclusions).

Keren and Boer (1985) suggested a third proposal to resolve the conflict between the RLIE and the FCE, a solution that referred to the knowledge of the target position. These authors were also able to demonstrate that the RLIE disappears, or even reverses, when participants can predict the target position. Together, the results of Keren and Boer's and our experiments show that the RLIE with simultaneous presentation of stimuli only arises when the participants do not know the target position in advance. In this situation, participants must identify both stimuli, because each of them could be the target. However, Keren and Boer assumed that two simultaneously presented stimuli are always processed in parallel, whether the target position is known in advance or not. Accordingly, these authors explained the disappearance of the RLIE with predictable target locations by assuming that participants focus attention on the target position in advance, which reduces or eliminates the effects of distractors on perceptual processing of the target. Yet this account is incompatible with the fact that with predictable target locations, identical distractors can produce redundancy gains that are - at least partly - attributed to interactions at a perceptual level (e.g., Fournier \& Eriksen, 1990; Taylor, 1977).

The discrepancies can only be resolved if we assume that predictability of the target location does not eliminate cross talk between distractor and target processing at perceptual levels, but only abolishes the necessity to identify the distractor. The necessity to identify both stimuli (i.e., distractor and target) with unpredictable target locations enforces serial identification in random order. Previous studies have revealed evidence that the identification of complex visual stimuli proceeds serially, by attending serially to stimulus positions (e.g., Treisman \& Gelade, 1980; Woodman \& Luck, 2003). With a predictable target location, however, distractors are processed in parallel with the target if the distractors are presented spatially close to the target. With parallel 
processing, identical distractors facilitate performance, whereas incompatible distractors impair performance (Experiment 3). However, because distractors are (almost) never completely identified before the target, RB does not occur in this situation. Accordingly, our account implies that the cause of RB must arise during a rather late stage of stimulus (i.e., distractor) processing, or even after stimulus identification has been completed.

Another interesting finding of the present study is that identical distractors caused substantial RB for target stimuli, even though the distractors never had to be reported. Even more, in Experiments 2 and 3, the distractors could have been completely ignored, because participants knew the constant target position in advance, and they were in fact explicitly told to ignore the distractor stimulus. Nevertheless, distractors preceding the target by not more than $280 \mathrm{msec}$ markedly impaired the identification of identical targets. This result suggests that even an irrelevant stimulus, which does not have to be processed at all, can cause RB for subsequently presented stimuli when the distractor is sufficiently salient and there is enough time to identify it. This conclusion is inconsistent with the - implicit or explicit-assumption of several authors that only stimuli that have to be identified and/or reported are tokenized (e.g., Epstein \& Kanwisher, 1999; Kanwisher, 1987).

In summary, the present study has revealed evidence contrary to the ideas that the RLIE is caused by parallel processing of two identical stimuli (Bjork \& Murray, 1977; Keren \& Boer, 1985) and is due to response strategies of the participants (C. W. Eriksen et al., 1981). Rather, the RLIE seems to arise from serial identification of two identical stimuli and, therefore, can be interpreted as an instance of RB. When processed in parallel with a target, identical stimuli produce redundancy gains, whereas incompatible stimuli produce response competition. Therefore, the present study has provided a step toward a better understanding of the mechanisms involved in producing cross talk between visual stimuli that are being processed concurrently.

Yet, open questions still remain. A first question is whether the present results could be replicated with nameable stimuli (e.g., letters) and vocal responses. A second relates to possible dissociations between speed and accuracy (cf. Johnston et al., 2002; Santee \& Egeth, 1982). For experimental conditions with predictable locations and simultaneous presentation of distractor and target, similar studies using RT as the main dependent variable exist, and these studies have revealed results similar to our accuracy results. For experimental conditions with unpredictable locations, however, comparable RT studies are lacking. We are currently working on resolving these issues.

\section{REFERENCES}

Anderson, C. J., \& Neill, W. T. (2002). Two Bs or not two Bs? A signal detection theory analysis of repetition blindness in a counting task. Perception \& Psychophysics, 64, 732-740.

Armstrong, I. T., \& Mewhort, D. J. K. (1995). Repetition deficit in rapid-serial-visual-presentation displays: Encoding failure or retrieval failure? Journal of Experimental Psychology: Human Perception \& Performance, 21, 1044-1052.

Bavelier, D., \& Potter, M. C. (1992). Visual and phonological codes in repetition blindness. Journal of Experimental Psychology: Human Perception \& Performance, 18, 134-147.

BJork, E. L., \& MurRaY, J. T. (1977). On the nature of input channels in visual processing. Psychological Review, 84, 472-484.

Chun, M. M. (1997). Types and tokens in visual processing: A double dissociation between the attentional blink and repetition blindness. Journal of Experimental Psychology: Human Perception \& Performance, 23, 738-755.

Egeth, H. E., \& Santee, J. L. (1981). Conceptual and perceptual components of interletter inhibition. Journal of Experimental Psychology: Human Perception \& Performance, 7, 506-517.

EPSTEIN, R., \& KANWISHER, N. (1999). Repetition blindness for locations: Evidence for automatic spatial coding in an RSVP task. Journal of Experimental Psychology: Human Perception \& Performance, 25, 1855-1866.

ERIKSEN, B. A., \& EriKsen, C. W. (1974). Effects of noise letters upon the identification of a target letter in a nonsearch task. Perception \& Psychophysics, 16, 143-149.

ERIKSEN, C. W., \& ERIKSEN, B. A. (1979). Target redundancy in visual search: Do repetitions of the target within the display impair processing? Perception \& Psychophysics, 26, 195-205.

Eriksen, C. W., Morris, N., Yeh, Y.-Y., O'Hara, W., \& Durst, R. T. (1981). Is recognition accuracy really impaired when the target is repeated in the display? Perception \& Psychophysics, 30, 375-385.

ERIKSEN, C. W., \& Schultz, D. W. (1979). Information processing in visual search: A continuous flow conception and experimental results. Perception \& Psychophysics, 25, 249-263.

EsTES, W. K. (1972). Interactions of signal and background variables in visual processing. Perception \& Psychophysics, 12, 278-286.

Estes, W. K. (1974). Redundancy of noise elements and signals in visual detection of letters. Perception \& Psychophysics, 16, 53-60.

Fagot, C., \& PAshler, H. (1995). Repetition blindness: Perception or memory failure? Journal of Experimental Psychology: Human Perception \& Performance, 21, 275-292.

Flowers, J. H., \& WILCOX, N. (1982). The effect of flanking context on visual classification: The joint contribution of interactions at different processing levels. Perception \& Psychophysics, 32, 581-591.

FoURNIER, L. R., \& ERIKSEN, C. W. (1990). Coactivation in the perception of redundant targets. Journal of Experimental Psychology: Human Perception \& Performance, 16, 538-550.

Johnston, J. C., Hochhaus, L., \& Ruthruff, E. (2002). Repetition blindness has a perceptual locus: Evidence from online processing of targets in RSVP streams. Journal of Experimental Psychology: Human Perception \& Performance, 28, 477-489.

Kahneman, D., Treisman, A., \& Burkell, J. (1983). The cost of visual filtering. Journal of Experimental Psychology: Human Perception \& Performance, 9, 510-522.

KANWISHER, N. G. (1987). Repetition blindness: Type recognition without token individuation. Cognition, 27, 117-143.

KANWISHER, N. G. (1991). Repetition blindness and illusory conjunctions: Errors in binding visual types with visual tokens. Journal of Experimental Psychology: Human Perception \& Performance, 17, 404-421.

Kanwisher, N. G., KIM, J. W., \& Wickens, T. D. (1996). Signal detection analyses of repetition blindness. Journal of Experimental Psychology: Human Perception \& Performance, 22, 1249-1260.

KANWISHER, N. G., \& PotTER, M. C. (1990). Repetition blindness: Levels of processing. Journal of Experimental Psychology: Human Perception \& Performance, 16, 30-47.

Keren, G., \& Boer, L. C. (1985). Necessary conditions for repeatedletter inferiority: The role of positional uncertainty. In M. I. Posner \& O. S. M. Marin (Eds.), Attention and performance XI (pp. 601612). Hillsdale, NJ: Erlbaum.

Kinchla, R. A. (1992). Attention. Annual Review of Psychology, 43, 711-742.

Luo, C. R., \& CARAmazza, A. (1995). Repetition blindness under minimum memory load: Effects of spatial and temporal proximity and 
the encoding effectiveness of the first item. Perception \& Psychophysics, 57, 1053-1064.

Luo, C. R., \& CARAmazza, A. (1996). Temporal and spatial repetition blindness: Effects of presentation mode and repetition lag on the perception of repeated items. Journal of Experimental Psychology: Human Perception \& Performance, 22, 95-113.

Miller, J. (1982). Divided attention: Evidence for coactivation with redundant signals. Cognitive Psychology, 14, 247-279.

Miller, J. (1991). The flanker compatibility effect as a function of visual angle, attentional focus, visual transients, and perceptual load: A search for boundary conditions. Perception \& Psychophysics, 49, 270-288.

Mozer, M. C. (1989). Types and tokens in visual letter perception. Journal of Experimental Psychology: Human Perception \& Performance, 15, 287-303.

Neill, W. T., Neely, J. H., Hutchison, K. A., Kahan, T. A., \& VerWys, C. A. (2002). Repetition blindness: Forward and backward. Journal of Experimental Psychology: Human Perception \& Performance, 28, 137-149.

PARK, J., \& KAnWISher, N. (1994). Determinants of repetition blind- ness. Journal of Experimental Psychology: Human Perception \& Performance, 20, 500-519.

SanteE, J. L., \& Egeth, H. E. (1980). Interference in letter identification: A test of feature-specific inhibition. Perception \& Psychophysics, 27, 321-330.

Santee, J. L., \& Egeth, H. E. (1982). Do reaction time and accuracy measure the same aspects of letter recognition? Journal of Experimental Psychology: Human Perception \& Performance, 8, 489-501.

TAYLOR, D. A. (1977). Time course of context effects. Journal of Experimental Psychology: General, 106, 404-426.

Treisman, A. M., \& Gelade, G. (1980). A feature-integration theory of attention. Cognitive Psychology, 12, 97-136.

Woodman, G. F., \& LUCK, S. J. (2003). Serial deployment of attention during visual search. Journal of Experimental Psychology: Human Perception \& Performance, 29, 121-138.

YanTis, S. (1993). Stimulus-driven attentional capture. Current Directions in Psychological Science, 2, 156-161.

(Manuscript received December 9, 2003;

revision accepted for publication August 30, 2004.) 